\title{
Comparing co-morbidities in total joint arthroplasty patients using the RxRisk-V, Elixhauser, and Charlson Measures: a cross-sectional evaluation
}

\author{
Maria C. S. Inacio ${ }^{1 *}$, Nicole L. Pratt ${ }^{1}$, Elizabeth E. Roughead ${ }^{1}$ and Stephen E. Graves ${ }^{2}$
}

\begin{abstract}
Background: Joint arthroplasty patients have a high prevalence of co-morbidities and this impacts their surgical outcomes. There are different ways to ascertain co-morbidities and appropriate measurement is necessary. The purpose of this study was to: (1) describe the prevalence of co-morbidities in a cohort of total hip arthroplasty (THA) and knee arthroplasty (TKA) patients using two diagnoses-based measures (Charlson and Elixhauser) and one prescription-based measure (RxRisk-V); (2) compare the agreement of co-morbidities amongst the measures.
\end{abstract}

Methods: A cross-sectional study of Australian veterans undergoing THAs $(n=11,848)$ and TKAs $(n=18,972)$ between 2001 and 2012 was conducted. Seventeen co-morbidities were identified using the Charlson, 30 using the Elixhauser, and 42 using the RxRisk-V measure. Agreement between co-morbidities was calculated using Kappa (K) statistics.

Results: Combining measures, 64 conditions were identified, of these 28 were only identified using the RxRisk-V, 11 using the Elixhauser, and 2 using the Charlson. The most prevalent conditions was pain treated with antiinflammatories (58.7\% THAs, $55.9 \%$ TKAs), pain treated with narcotics (55.0\% THAs, $50.9 \%$ TKAs), hypertension (56.0 \% THAs and TKAs), and anticoagulation disorders (53.0 \% THAs, $48.6 \%$ TKAs). Diabetes was the only condition with substantial agreement (all $\mathrm{k}>0.6$ ) amongst all measures. When comparing the diagnoses based algorithms, agreement was high for overlapping conditions (all $\mathrm{k}>0.71$ ).

Conclusions: Different measures identified different co-morbidities, provided different estimates for the same co-morbidity, and had different levels of agreement for common co-morbidities. This highlights the importance of understanding co-morbidity measures and using them appropriately in studies and case-mix adjustments analyses.

Keywords: Co-morbidities, Total joint arthroplasty, Pharmacy data, RxRisk-V, Charlson, Elixhauser

\section{Background}

The prevalence of multi-morbidity is increasing [1, 2], thus the number of patients with multiple co-morbidities undergoing total joint arthroplasty has also increased in the last two decades $[3,4]$. Studies in the United States (US) report multiple co-morbidities in $62 \%$ of patients over 65 years old and this was associated with higher healthcare utilisation, cost, and overall impairment of

\footnotetext{
* Correspondence: maria.inacio@unisa.edu.au

'Quality Use of Medicines and Pharmacy Research Centre, Medicine and Device Surveillance Centre of Research Excellence, Sansom Institute, School of Pharmacy and Medical Sciences, University of South Australia, GPO Box 2471, Adelaide 5001, South Australia, Australia

Full list of author information is available at the end of the article
}

patients [2]. In the US in the mid-2000s, patients older than 65 years undergoing total joint arthroplasty had an average of two co-morbidities when measured by the diagnosis based Elixhauser co-morbidity measure [3, 4]. The number of co-morbidities as well as specific conditions are related to patients' surgical outcome during total joint arthroplasty [5-12].

Appropriate measurement of patient co-morbidities in those undergoing joint arthroplasty is critical because of the high prevalence of co-morbidities, the higher service utilisation, and the poorer surgical outcomes in those patients. Several co-morbidity measures exist [13-15]. The validated and most frequently used diagnostic based 
coding measure have been shown to be predictive of several healthcare outcomes in different study populations (including orthopaedics and other disciplines) and are used in case mix adjustment $[15,16]$. Two of the most commonly used co-morbidity measures in health services and orthopaedics research are the Charlson [17] and Elixhauser [18] measures. Both measures have been used in studies evaluating total joint arthroplasty patients. Prescription medication based co-morbidity measures, such as the RxRisk-V [19] and Medication Based Disease Burden Index [20], were also developed to estimate the prevalence of co-morbidities in different populations and have been used in case mix adjustment and outcome prediction. However, to our knowledge, no previous study has evaluated the prevalence of co-morbidities in a total joint arthroplasty sample using a validated prescriptionbased coding measure.

Each method to ascertain co-morbidities has different strengths and limitations. For example, pharmacy based measures may provide more detailed information on active chronic conditions for which patients are actually receiving care and not only serious conditions that diagnostic based measures may identify. Further, difference in coding practices between countries may make one measure more relevant in a certain country. In Australia, for example, the use of the diagnoses based measures is limited to inpatient encounters as outpatient encounters are not recorded using the same coding system necessary for identification of the conditions. Additionally, in Australia and the US, hospital coding does not have an official mechanism to include diagnoses other than those relevant to the hospital stay [21]. This is probably avoided by countries such as the United Kingdom, which implemented in 2010 a list of required comorbidities that must be reported at the time of hospitalisation [15].

It is clinically and scientifically important to have an accurate, comprehensive, and standardized method to identify the presence of co-morbidities at the time of surgery for several reasons. One reason is to inform surgeons, healthcare workers, and patients on the proper course of treatment and risk of procedure, especially when multiple co-morbidities are present. Another is for standardizing research, specifically when evaluating outcomes in different cohorts of patients where proper case mix adjustment must be conducted to assure cohorts can be properly compared. Our study sought to (1) determine the prevalence of co-morbidities in an Australian cohort of total hip arthroplasty (THA) and total knee arthroplasty (TKA) patients using two diagnoses based comorbidity measures (Charlson [17] and Elixhauser [18]) and one prescription based measure (RxRisk-V [19, 22]) and (2) compare the agreement of individual comorbidities amongst the measures.

\section{Methods}

\section{Study design, setting, and sample}

A cross-sectional study was conducted on a cohort of THA and TKA patients, who had their procedures between 2001 and 2012. Patients receiving care subsidised by the Australian Government Department of Veterans' Affairs (DVA) were included in the study. Deidentified administrative inpatient encounter information and prescription medicine (inpatient and outpatient) data for this captured population was used.

The study sample included patients aged $\geq 18$ years old, who had all health services subsidised by DVA, and underwent elective primary unilateral THA procedures identified using International Classification of Disease, $10^{\text {th }}$ Revision, Australian Modification (ICD-10-AM) code 4931800 and elective primary unilateral TKA procedures identified using ICD-10-AM codes 4951800, 4952100, 4952102, 4952400.

\section{Co-morbidity measures and data sources}

The RxRisk-V [19] is a co-morbidity prescription based measure that uses patients' medication histories to determine the presence of 45 conditions [22]. This measure has been shown to be predictive of cost of care $[19,22]$ and mortality $[23-25]$ in different patient samples using both inpatient and outpatient pharmacy data $[23,25]$. In this study a modified RxRisk-V was used with 42 conditions; the conditions ostomy, neurogenic bladder, and urinary incontinence were excluded.

The Charlson co-morbidity measure typically uses inpatient hospitalisations over a specified time period to identify the presence of 17 conditions and calculate an overall comorbidity score $[21,26]$. The Charlson score was originally developed to predict mortality and assist with case mix adjustment in regards to this outcome, but has been applied to several other outcomes now, including some surgical outcomes [16, 27].

The Elixhauser co-morbidity measure also typically uses inpatient hospitalisations during a specific period to calculate co-morbidities. The most common form of this measure identifies the presence of 30 conditions and has been evaluated as a predictor of blood transfusions, length of stay, and mortality $[26,28]$. This measure was developed by the Agency for Healthcare Research and Quality (AHRQ) Healthcare Cost and Utilisation Project and is widely used in health services research $[18,29]$.

The RxRisk-V and Charlson have 6 common conditions, the Elixhauser and RxRisk-V have 10 common conditions, and the Charlson and Elixhauser have 12.

Using the DVA administrative database all inpatient hospitalisations and prescription medicine history were identified for the study sample. The database contains details of all prescription medications, medical, allied health services and hospitalisations provided to veterans 
for which DVA pays a subsidy. In the dataset, medications are coded according to the World Health Organization Anatomic, Therapeutic and Chemical Classification, and the Pharmaceutical Benefits Schedule item codes. Hospitalisations are coded according to the ICD-10-AM. DVA also maintains a client file, which contains information on gender, date of birth, date of death, and family status for a treatment population that in September 2011 was 242,000 people.

In this study, the 12 month period preceding the discharge date of the arthroplasty procedure was used to ascertain the co-morbidities according to the two diagnoses based co-morbidity measures (Charlson and Elixhauser) using DVA hospital records. The arthroplasty procedure hospitalisation was included in the calculation of the diagnostic co-morbidity measures. The 12 month prescription dispensing history preceding the admission date for the arthroplasty procedure was utilised to measure RxRisk-V.

\section{Statistical analysis}

Frequencies, proportions, means, standard deviations (SD), medians and interquartile ranges (IRQs) were used to describe the sample. Prevalence of co-morbidities by each measure was calculated. Agreement between specific co-morbidity indicators between measures (where conditions were common) was calculated using Kappa ( $\mathrm{k}$ ) statistics and $95 \%$ confidence intervals $(\mathrm{CI})$. $\mathrm{k}$ agreement thresholds used were: slight: $0.01 \leq \kappa \leq 0.20$, fair: $0.21 \leq \kappa \leq$ 0.40, moderate: $0.41 \leq \kappa \leq 0.60$, substantial: $0.61 \leq \kappa \leq 0.80$, almost perfect: $0.80 \leq \kappa \leq 0.99$, perfect: $\kappa=1.0$ [30]. SAS 9.4 (SAS Institute, Cary, NC, USA) was used for all analyses.

This study has ethics approval from the Australian DVA and University of South Australia human research ethics committees. The ethics committees also waived the requirement for informed consent.

\section{Results}

During the study period 11,848 patients underwent THA and 18,972 TKAs. Both cohorts had a slightly higher proportion of males (50.2 \% THA and $52.3 \%$ TKA) than females and procedures were mostly performed in private hospitals (95.6 \% THA and $96.6 \%$ TKA). The median age was 80.9 (IQR 76.4-84.4) years old for patients with THAs and 79.8 (IQR $=74.7-83.5)$ years old for patients with TKAs. See Table 1 for sample details.

The mean number of RxRisk-V, Elixhauser, and Charlson co-morbidities in the THA (5.5 ( $\mathrm{SD}=3.3), 0.9$ $(\mathrm{SD}=1.3), 0.4 \quad(\mathrm{SD}=0.8)$, respectively) and TKA (5.4 ( $\mathrm{SD}=3.5), 0.9(\mathrm{SD}=1.2), 0.4$ ( $\mathrm{SD}=0.7)$, respectively) cohorts was similar. The three most common co-morbidities identified by the RxRisk-V were also similar between the THA and TKA cohort, and included: the musculoskeletal conditions of pain treated with anti-inflammatories
(58.7 \% THA, $55.9 \%$ TKA) and pain treated with narcotics (55.0 \% THA, $50.9 \%$ TKA), and cardiovascular diseases that involved treatment with anticoagulation agents (52.6 \% THA, 48.4 \% TKA). Using the Elixhauser measure, the three most prevalent co-morbidities in patients having a THA were: hypertension (22.0 \%), arrhythmias (14.7\%), and fluid and electrolyte disorders (7.5\%); in patients with TKAs the conditions were: hypertension (23.4\%), arrhythmias (13.5\%), and diabetes with chronic complications (7.7 \%). Using the Charlson measure the three more prevalent co-morbidities of THA and TKA patients were: uncomplicated diabetes $(7.1 \%$ THA, $8.1 \%$ TKA), diabetes with chronic complications $(6.3 \%$ THA, $7.3 \%$ TKA), and chronic pulmonary disease (5.5\% THA, $4.5 \%$ TKA). See Table 2 for co-morbidities by the three measures and overall prevalence of conditions.

Combining all measures, 64 unique co-morbidities were identified, of these 28 were only identified using the RxRiskV, 11 using the Elixhauser, and 2 using the Charlson. The most prevalent co-morbidities only identified by the RxRisk-V included: pain treated with anti-inflammatories, pain treated with narcotics, gastric acid disorder, hyperlipidaemia, ischemic heart disease (both in combination with angina and hypertension), diseases treated with antiplatelets agents, and reactive airway disease. The most prevalent co-morbidities only identified by the Elixhauser included fluid and electrolyte disorders, obesity, valvular disease, history of solid tumour, deficiency anaemia, and obesity. The co-morbidities only identified by Charlson were cerebrovascular disease and myocardial infarction. See Table 2 other co-morbidities specific to each measure.

There was a relationship between co-morbidity measures for common indicators (Table 3). RxRisk-V and the Elixhauser, diabetes was the only co-morbidity with substantial agreement between the two measures $(\mathrm{K}=0.63,95 \%$ CI 0.60-0.65 for THA, $\mathrm{k}=0.61,95 \%$ CI 0.59-0.63 for TKA). Similarly, when comparing the RxRisk-V and Charlson measures, diabetes was also the only co-morbidity with substantial agreement $((\kappa=0.63,95 \%$ CI $0.60-0.65$ for THAs, $\mathrm{K}=0.61,95 \%$ CI 0.59-0.63 for TKAs). When comparing the two diagnostic based measures, the Charlson and Elixhauser, for conditions that did not have identical coding, the agreement was almost perfect for uncomplicated diabetes, diabetes with chronic complications, peptic ulcer disease, rheumatoid arthritis, and renal failure. Mild liver disease had substantial agreement between the Charlson and Elixhauser $(\mathrm{K}=0.71,95 \%$ CI $0.56-0.85$ for THA and $\kappa=0.76,95 \%$ CI 0.67-0.86 for TKA).

\section{Discussion}

The co-morbidity measures evaluated in this study yielded a different prevalence of co-morbidities and a wider variation of agreement between common conditions. In our sample of joint arthroplasty patients, the most 
Table 1 Total hip and knee arthroplasty patients characteristics, 2001-2012

\begin{tabular}{|c|c|c|c|c|c|}
\hline & & \multicolumn{2}{|c|}{ Total hip arthroplasty } & \multicolumn{2}{|c|}{ Total knee arthroplasty } \\
\hline & & $\mathrm{N}$ & (\%) & $\mathrm{N}$ & $(\%)$ \\
\hline Total & & 11,848 & 100.0 & 18,972 & 100.0 \\
\hline \multirow[t]{2}{*}{ Gender } & Females & 5,898 & 49.8 & 9,047 & 47.7 \\
\hline & Males & 5,950 & 50.2 & 9,925 & 52.3 \\
\hline Age, years (median, IQR) & & 80.9 & $76.4-84.4$ & 79.8 & $74.7-83.5$ \\
\hline \multirow[t]{4}{*}{ THA diagnoses (ICD-10-AM code) } & Other primary coxarthrosis (M161) & 9,648 & 81.4 & - & - \\
\hline & Coxarthrosis unspecified (M169) & 1,109 & 9.4 & - & - \\
\hline & Unspecified osteonecrosis pelvis thigh (M8795) & 343 & 2.9 & - & - \\
\hline & Other & 748 & 6.3 & & \\
\hline \multirow[t]{4}{*}{ TKA Diagnosis (ICD-10-AM Code) } & Other primary gonarthrosis (M171) & - & - & 16,329 & 86.1 \\
\hline & Gonarthrosis unspecified (M179) & - & - & 1,437 & 7.6 \\
\hline & Primary gonarthrosis bilateral (M170) & - & - & 489 & 2.6 \\
\hline & Other & - & - & 717 & 3.8 \\
\hline \multirow[t]{5}{*}{ Number of RxRisk-V co-morbidities } & 0 & 1,466 & 12.4 & 3,290 & 17.3 \\
\hline & $1-2$ & 747 & 6.3 & 938 & 4.9 \\
\hline & $3-4$ & 2,041 & 17.2 & 2,785 & 14.7 \\
\hline & $5-6$ & 2,888 & 24.4 & 4,398 & 23.2 \\
\hline & $\geq 7$ & 4,706 & 39.7 & 7,561 & 39.9 \\
\hline \multirow[t]{3}{*}{ Number of Elixhauser co-morbidities } & 0 & 6,087 & 51.4 & 9,910 & 52.2 \\
\hline & $1-2$ & 4,333 & 12.1 & 7,034 & 37.1 \\
\hline & $\geq 3$ & 1,428 & 12.1 & 2,028 & 10.7 \\
\hline \multirow[t]{3}{*}{ Number of Charlson co-morbidities } & 0 & 8,529 & 72.0 & 13,917 & 73.4 \\
\hline & $1-2$ & 2,946 & 24.9 & 4,628 & 24.4 \\
\hline & $\geq 3$ & 373 & 3.1 & 427 & 2.3 \\
\hline
\end{tabular}

IQR interquartile range, THA total hip arthroplasty, TKA total knee arthroplasty, ICD-10-AM International Classifications of Disease, 10th Revision, Australian Modification

common non-musculoskeletal related co-morbidities were hypertension, disorders that required anticoagulation agents, and gastric acid disorders. We also found that except for diabetes, which had good agreement between all three co-morbidity measures, there was low agreement between the prescription based RxRisk- $\mathrm{V}$ and the diagnoses based Elixhauser and Charlson co-morbidity measures for common conditions. As expected, between the diagnoses based measures there was substantial agreement between common co-morbidities.

The prevalence of co-morbidities identified using all three measurements had some commonalties and differences with previous estimates in large cohorts of joint arthroplasty patients. Some of the differences are attributed to the method of co-morbidity ascertainment in different studies. The Elixhauser was the more commonly used measure in the published literature, such as Cram et al.'s US Medicare studies [3, 4], Kapoor et al.'s US DVA study [31], the Kaiser Permanente Total Joint Replacement registry studies [32, 33], and the US California and New York states cohort studies by Dy et al. [34, 35]. But other studies have used different measures, such as an US
Medicare TKA study that used a combination of the Charlson and Elixhauser [5], and a Finish arthroplasty registry study that used a non validated measure inclusive of diagnostic codes, medication prescriptions, and drug reimbursement for certain conditions [36]. Most studies reported similar high prevalence of hypertension (range $43-70 \%$ ) [5, 31-35], but the Finish study reported a lower prevalence (17.7\% in THA and $20.8 \%$ in TKA) than these cohorts and ours [36]. The prevalence of diabetes was also similar between our cohort (approximately 10-13 \%) and those of the US California and New York states studies (9 \% in the THAs and $14 \%$ in TKAs), US Medicare THA cohort (10-15\% recent estimates) and the Finish arthroplasty registry cohort $(5.5-7.5 \%)[3,35,36]$. This prevalence was lower, however, than those reported by other US cohorts (Kaiser Permanente registry 18-26\%, Medicare TKA patients $22 \%$, and DVA patients $26.5 \%$ ) $[4,5,31-33]$. The Kaiser Permanente registry ascertained diabetes using the institution's diabetes registry, instead of the Elixhauser, which could contribute to the higher prevalence reported [37]. The prevalence of congestive heart failure (approximately $13 \%$ ) was also similar to that 
Table 2 Mean total scores and prevalence of individual conditions by each co-morbidity measure and total computation

\begin{tabular}{|c|c|c|c|c|c|c|c|c|}
\hline & \multicolumn{4}{|c|}{ Total hip arthroplasty $(N=11,848)$} & \multicolumn{4}{|c|}{ Total knee arthroplasty $(N=18,972)$} \\
\hline & RxRisk-V & Elixhauser & Charlson & Total & RxRisk-V & Elixhauser & Charlson & Total \\
\hline \multirow[t]{2}{*}{ Mean score (SD) } & $5.5(3.3)$ & $0.9(1.3)$ & $0.4(0.8)$ & $6.2(3.6)$ & $5.4(3.5)$ & $0.9(1.2)$ & $0.4(0.7)$ & $6.1(3.7)$ \\
\hline & N (\%) & N (\%) & N (\%) & N (\%) & N (\%) & N (\%) & N (\%) & N (\%) \\
\hline \multicolumn{9}{|l|}{ Cancer } \\
\hline Lymphoma & - & $42(0.4)$ & - & $42(0.4)$ & - & $33(0.2)$ & - & $33(0.2)$ \\
\hline Malignancies & $423(3.6)$ & & $257(2.2)$ & $648(5.5)$ & $757(4.0)$ & & $285(1.5)$ & $1,004(5.3)$ \\
\hline Metastatic cancer & - & $60(0.5)$ & $60(0.5)$ & $60(0.5)$ & - & $43(0.2)$ & $43(0.2)$ & $43(0.2)$ \\
\hline Solid tumor without metastatis & - & $303(2.6)$ & - & $306(2.6)$ & - & $398(2.1)$ & - & $398(2.1)$ \\
\hline \multicolumn{9}{|l|}{ Cardiovascular/blood } \\
\hline Anticoagulation agents/coagulopathy & $6,230(52.6)$ & $130(1.1)$ & - & $6,274(53.0)$ & $9,177(48.4)$ & $168(0.9)$ & - & $9,227(48.6)$ \\
\hline Antiplatelets agents & $3,975(33.5)$ & - & - & $3,975(33.5)$ & $6,055(31.9)$ & - & - & $6,055(31.9)$ \\
\hline Arrhythmias & $1,206(10.2)$ & $1,744(14.7)$ & - & $2,313(19.5)$ & $1,766(9.3)$ & $2,561(13.5)$ & - & $3,427(18.1)$ \\
\hline Cerebrovascular disease & - & - & $273(2.3)$ & $273(0.3)$ & - & - & $353(1.9)$ & $353(1.8)$ \\
\hline Congestive heart failure & $1,454(12.3)$ & $471(4.0)$ & $471(4.0)$ & $1,636(13.8)$ & $2,142(11.3)$ & $608(3.2)$ & $608(3.2)$ & $2,452(12.9)$ \\
\hline Hyperlipidaemia & $4,377(36.9)$ & - & - & $4,377(36.9)$ & $7,115(37.5)$ & - & - & $7,115(37.5)$ \\
\hline Hypertension $^{\mathrm{a}}$ & $5,644(47.6)$ & $2,612(22.0)$ & - & $6,638(56.0)$ & $8,866(46.7)$ & 4,433 (23.4) & - & $10,629(56.0)$ \\
\hline Ischemic heart disease/angina & $1,375(11.6)$ & - & - & $1,375(11.6)$ & $2,054(10.8)$ & - & - & $2,054(10.8)$ \\
\hline Ischemic heart disease/hypertension & $4,054(34.2)$ & - & - & $4,054(34.2)$ & $6,329(33.4)$ & - & - & $6,329(33.4)$ \\
\hline Myocardial infarction & - & - & $333(2.8)$ & $333(2.8)$ & - & - & $410(2.2)$ & $410(2.2)$ \\
\hline Peripheral vascular disease & - & $299(2.5)$ & $299(2.5)$ & $299(2.5)$ & - & $346(1.8)$ & $346(1.8)$ & $346(1.8)$ \\
\hline Pulmonary circulation disorders & - & $136(1.1)$ & - & $136(1.2)$ & - & $288(1.5)$ & - & $288(1.5)$ \\
\hline Valvular disease & - & $308(2.6)$ & - & $308(2.6)$ & - & $414(2.2)$ & - & $414(2.2)$ \\
\hline \multicolumn{9}{|l|}{ Endocrine } \\
\hline Diabetes (uncomplicated) & $905(7.6)$ & $755(6.4)$ & $838(7.1)$ & $1,271(10.7)$ & $1,782(9.4)$ & $1,405(7.4)$ & $1,530(8.1)$ & $2,423(12.8)$ \\
\hline Diabetes (complicated) & - & $794(6.7)$ & $747(6.3)$ & $794(6.7)$ & - & $1,452(7.7)$ & $1387(7.3)$ & $1,452(7.7)$ \\
\hline Hypothyroidism & $788(6.7)$ & $67(0.6)$ & - & $801(6.8)$ & $1339(7.1)$ & $95(0.5)$ & - & $1,369(7.2)$ \\
\hline Pancreatic insufficiency & $20(0.2)$ & - & - & $20(0.2)$ & $31(0.2)$ & - & - & $31(0.2)$ \\
\hline \multicolumn{9}{|l|}{ Gastrointestinal } \\
\hline Gastric acid disorder & $5,307(44.8)$ & - & - & $5,307(44.8)$ & $8,436(44.5)$ & - & - & $8,436(44.5)$ \\
\hline Inflammatory bowel syndrome & $118(1.0)$ & - & - & $118(1.0)$ & $180(1.0)$ & - & - & $180(1.0)$ \\
\hline Hepatitis C & $0(0.0)$ & - & - & $0(0.0)$ & $0(0.0)$ & - & - & $0(0.0)$ \\
\hline Liver disease (mild) & - & $25(0.2)$ & $26(0.2)$ & $33(0.3)$ & - & $42(0.2)$ & $47(0.2)$ & $55(0.3)$ \\
\hline Liver disease (severe) or failure & $479(4.0)$ & - & $11(0.1)$ & $487(4.1)$ & $542(2.9)$ & - & $9(<0.01)$ & $551(2.9)$ \\
\hline Peptic ulcer disease & - & $83(0.7)$ & $119(1.0)$ & $119(1.0)$ & - & $102(0.5)$ & $155(0.8)$ & $155(0.8)$ \\
\hline \multicolumn{9}{|l|}{ Muscuoskeletal/pain related } \\
\hline Gout & $1,187(10.0)$ & - & - & $1,187(10.0)$ & $2,210(11.6)$ & - & - & $2,210(11.7)$ \\
\hline Migraine & $37(0.3)$ & - & - & $37(0.3)$ & $104(0.5)$ & - & - & $104(0.6)$ \\
\hline Osteoporosis/Pagets & $1,463(12.3)$ & - & - & $1,463(12.3)$ & $2,017(10.6)$ & - & - & $2,017(10.6)$ \\
\hline Pain & $6,512(55.0)$ & - & - & $6,512(55.0)$ & $9,663(50.9)$ & - & - & $9,663(50.9)$ \\
\hline Pain/Inflammation & $6,958(58.7)$ & - & - & $6,958(58.7)$ & $10,611(55.9)$ & - & - & $10,611(55.9)$ \\
\hline RA/collage vascular disorders & - & $197(1.7)$ & $176(1.5)$ & $197(1.7)$ & - & $348(1.8)$ & $320(1.7)$ & $349(1.8)$ \\
\hline
\end{tabular}


Table 2 Mean total scores and prevalence of individual conditions by each co-morbidity measure and total computation (Continued)

\begin{tabular}{|c|c|c|c|c|c|c|c|c|}
\hline \multicolumn{9}{|l|}{ Neurologic } \\
\hline Dementia & $92(0.8)$ & - & $209(1.8)$ & $249(2.1)$ & $94(0.5)$ & - & $195(1.0)$ & $237(1.3)$ \\
\hline Epilepsy & $535(4.5)$ & - & - & $535(4.5)$ & $838(4.4)$ & - & - & $838(4.4)$ \\
\hline Paralysis (or Paraplegia/Hemiplegia) & - & $86(0.7)$ & $86(0.7)$ & $86(0.7)$ & - & $112(0.6)$ & $112(0.6)$ & $112(0.6)$ \\
\hline Parkinson's disease & $198(1.7)$ & - & - & $198(1.7)$ & $367(1.9)$ & - & - & $367(1.9)$ \\
\hline Other neurological disorders & - & $155(1.3)$ & - & $155(1.3)$ & - & $205(1.1)$ & - & $205(1.1)$ \\
\hline \multicolumn{9}{|l|}{ Nutritional/obesity } \\
\hline Blood loss anaemia & - & $84(0.7)$ & - & $84(0.7)$ & - & $67(0.4)$ & - & $67(0.4)$ \\
\hline Deficiency anaemia & - & $203(1.7)$ & - & $203(1.7)$ & - & $223(1.2)$ & - & $223(1.2)$ \\
\hline Fluid and electrolyte disorders & - & $890(7.5)$ & - & $890(7.5)$ & - & $1,095(5.8)$ & - & $1,095(5.8)$ \\
\hline Hyperkalaemia & $8(0.1)$ & - & - & $8(0.1)$ & $18(0.1)$ & - & - & $18(0.1)$ \\
\hline Malnutrition & $22(0.2)$ & - & - & $22(0.2)$ & $37(0.2)$ & - & - & $37(0.2)$ \\
\hline Obesity & - & $194(1.6)$ & - & $194(1.6)$ & - & $500(2.6)$ & - & $500(2.6)$ \\
\hline Weight loss & - & $123(1.0)$ & - & $123(1.0)$ & - & $99(0.5)$ & - & $99(0.5)$ \\
\hline \multicolumn{9}{|l|}{ Psychological/behavioural } \\
\hline Alcohol abuse/dependence & $11(0.1)$ & $113(1.0)$ & - & $120(1.0)$ & $27(0.1)$ & $177(0.9)$ & - & $193(1.0)$ \\
\hline Anxiety and tension & $1,459(12.3)$ & - & - & $1,459(12.3)$ & $2,344(12.3)$ & - & - & $2,340(12.3)$ \\
\hline Bipolar disorder & $30(0.3)$ & - & - & $30(0.3)$ & $49(0.3)$ & - & - & $49(0.3)$ \\
\hline Depression & 2,598 (21.9) & $213(1.8)$ & - & $2,667(22.5)$ & $4,484(23.6)$ & $293(1.5)$ & - & $4,567(24.1)$ \\
\hline Drug abuse & & $14(0.1)$ & - & $14(0.1)$ & & $20(0.1)$ & - & $20(0.1)$ \\
\hline Psychotic illness/psychoses & $250(2.1)$ & $16(0.1)$ & - & $259(2.2)$ & $391(2.1)$ & $22(0.1)$ & - & $404(2.1)$ \\
\hline Smoking cessation & $102(0.9)$ & - & - & $102(0.9)$ & $159(0.8)$ & - & - & $159(0.8)$ \\
\hline \multicolumn{9}{|l|}{ Renal/urologic } \\
\hline Benign prostatic hypertrophy & $445(3.8)$ & - & - & $445(3.8)$ & $731(3.9)$ & - & - & $731(3.9)$ \\
\hline Renal disease/failure & $162(1.4)$ & $436(3.7)$ & $388(3.3)$ & $560(4.7)$ & $290(1.5)$ & $575(3.0)$ & $524(2.8)$ & $826(4.4)$ \\
\hline \multicolumn{9}{|l|}{ Respiratory } \\
\hline Chronic pulmonary disease & - & $652(5.5)$ & $651(5.5)$ & $652(5.5)$ & - & $858(4.5)$ & $856(4.5)$ & $858(4.5)$ \\
\hline Reactive airway disease & 2,461 (20.8) & - & - & $2,461(20.8)$ & $3,815(20.1)$ & - & - & $3,815(20.1)$ \\
\hline Tuberculosis & $0(0.0)$ & - & - & $0(0.0)$ & $1(<0.01)$ & - & - & $1(<0.01)$ \\
\hline \multicolumn{9}{|l|}{ Miscellaneous } \\
\hline Allergies & $1,660(14.0)$ & - & - & $1,660(14.0)$ & ,3046 (16.1) & - & - & $3,046(16.1)$ \\
\hline Glaucoma & $1,091(9.2)$ & - & - & $1,091(9.2)$ & $1,642(8.7)$ & - & - & $1,642(8.7)$ \\
\hline HIV/AIDS & $0(0.0)$ & $0(0.0)$ & $0(0.0)$ & $0(0.0)$ & $0(0.0)$ & $0(0.0)$ & $0(0.0)$ & $0(0.0)$ \\
\hline Psoriasis & $63(0.5)$ & - & - & $63(0.5)$ & $103(0.5)$ & - & - & $103(0.5)$ \\
\hline Steroid-responsive conditions & $1,889(15.9)$ & - & - & $1,889(15.9)$ & $3,345(17.6)$ & - & - & 3,345 (17.6) \\
\hline Transplant & $3(<0.01)$ & - & - & $3(<0.01)$ & $3(<0.01)$ & - & - & $3(<0.01)$ \\
\hline
\end{tabular}

$S D$ standard deviation, $C I$ confidence interval, $R A$ rheumatoid arthritis, HIV/AIDS human immunodeficiency virus/Acquired immune deficiency virus ${ }^{a}$ Complicated and uncomplicated combined

reported by one study reporting on the US TKA Medicare cohort (10\%) [6], but higher than all other studies reviewed (range 2.6-5.2 \%) [3, 4, 31-36]. Conversely, the prevalence of chronic obstructive pulmonary disease in our sample (5-6\%) was lower than that reported by almost all cohorts (range 11-18 \%) [6, 31, 34, 35] but the Finish arthroplasty register cohort (6-8 \%) [36]. While there are obvious differences in the cohorts of patients in these studies, disease prevalence differences are also due to how they were identified- highlighting the need for consideration in how co-morbidities are determined.

There was only a strong agreement between the comorbidities identified by the Risk- $\mathrm{V}$ and the Elixhauser (10 common conditions) and Charlson (6 common 
Table 3 Agreement between each co-morbidity measure of individual conditions. Cohen's Kappa coefficient and $95 \%$ confidence intervals for agreement estimations

\begin{tabular}{|c|c|c|c|c|c|c|}
\hline & \multicolumn{3}{|c|}{ Total hip arthroplasty } & \multicolumn{3}{|c|}{ Total knee arthroplasty } \\
\hline & $\begin{array}{l}\text { RxRisk-V } \\
\text { Elixhauser }^{\mathrm{a}}\end{array}$ & $\begin{array}{l}\text { RxRisk-V } \\
\text { Charlson }\end{array}$ & $\begin{array}{l}\text { Elixhauser } \\
\text { Charlson }^{c}\end{array}$ & $\begin{array}{l}\text { RxRisk-V } \\
\text { Elixhauser }^{\text {a }}\end{array}$ & $\begin{array}{l}\text { RxRisk-V } \\
\text { Charlson }^{b}\end{array}$ & $\begin{array}{l}\text { Elixhauser } \\
\text { Charlson }^{c}\end{array}$ \\
\hline & k $(95 \%$ Cl) & k $(95 \%$ Cl) & k $(95 \% \mathrm{Cl})$ & k $(95 \% \mathrm{Cl})$ & $\mathrm{k}(95 \% \mathrm{Cl})$ & k $(95 \%$ Cl) \\
\hline \multicolumn{7}{|l|}{ Cancer } \\
\hline Malignancies & - & $0.07(0.04-0.10)$ & - & - & $0.06(0.03-0.08)$ & - \\
\hline Metastatic cancer & & - & $1.00(1.00-1.00)$ & & - & $1.00(1.00-1.00)$ \\
\hline \multicolumn{7}{|l|}{ Cardiovascular/blood } \\
\hline Anticoagulation agents/coagulopathy & $0.01(0.00-0.01)$ & & & $0.00(0.00-0.01)$ & & \\
\hline Arrhythmias & $0.35(0.33-0.38)$ & - & - & $0.34(0.32-0.36)$ & - & - \\
\hline Congestive heart failure & $0.26(0.23-0.28)$ & $0.26(0.23-0.28)$ & $1.00(1.00-1.00)$ & $0.18(0.16-0.20)$ & $0.18(0.16-0.20)$ & $1.0(1.00-1.00)$ \\
\hline $\begin{array}{l}\text { Hypertension (complicated and } \\
\text { uncomplicated) }\end{array}$ & $0.13(0.11-0.14)$ & - & - & $0.13(0.11-0.14)$ & - & - \\
\hline Peripheral vascular disease & - & - & $1.00(1.00-1.00)$ & - & - & $1.00(1.00-1.00)$ \\
\hline \multicolumn{7}{|l|}{ Endocrine } \\
\hline Diabetes (uncomplicated) & $0.63(0.60-0.65)$ & $0.63(0.60-0.65)$ & $0.94(0.93-0.96)$ & $0.61(0.59-0.63)$ & $0.61(0.59-0.63)$ & $0.95(0.95-0.96)$ \\
\hline Diabetes (complicated) & - & - & $0.97(0.96-0.98)$ & - & - & $0.98(0.97-0.98)$ \\
\hline Hypothyroidism & $0.12(0.09-0.15)$ & - & - & $0.08(0.06-0.10)$ & - & - \\
\hline \multicolumn{7}{|l|}{ Gastrointestinal } \\
\hline Liver disease (mild) & - & & $0.71(0.56-0.85)$ & - & - & $0.76(0.67-0.86)$ \\
\hline Liver disease (severe) or failure & - & $0.01(0.00-0.02)$ & - & - & $0.00(0.00-0.00)$ & - \\
\hline Peptic ulcer disease & - & - & $0.82(0.76-0.88)$ & - & - & $0.79(0.74-0.85)$ \\
\hline \multicolumn{7}{|l|}{ Muscuoskeletal/pain related } \\
\hline $\begin{array}{l}\text { Rheumatoid arthritis/collage } \\
\text { vascular disorders }\end{array}$ & - & - & $0.94(0.92-0.97)$ & - & - & $0.95(0.94-0.97)$ \\
\hline \multicolumn{7}{|l|}{ Neurologic } \\
\hline Dementia & - & $0.34(0.27-0.41)$ & - & - & $0.36(0.28-4.43)$ & - \\
\hline Paralysis (or Paraplegia/Hemiplegia) & - & - & $1.00(1.00-1.00)$ & - & - & $1.00(1.00-1.00)$ \\
\hline \multicolumn{7}{|l|}{ Psychological/behavioral } \\
\hline Alcohol abuse/dependence & $0.06(0.00-0.12)$ & - & - & $0.10(0.05-0.16)$ & - & - \\
\hline Depression & $0.07(0.06-0.08)$ & - & & $0.06(0.05-0.07)$ & - & \\
\hline Psychotic illness/psychoses & $0.05(0.01-0.09)$ & - & & $0.04(0.01-0.07)$ & - & \\
\hline \multicolumn{7}{|l|}{ Renal/urologic } \\
\hline Renal disease/failure & $0.11(0.07-0.15)$ & $0.11(0.08-0.15)$ & $0.94(0.92-0.96)$ & $0.07(0.04-0.10)$ & $0.07(0.05-0.10)$ & $0.95(0.94-0.97)$ \\
\hline
\end{tabular}

Respiratory

Chronic pulmonary disease

$1.00(1.00-1.00)$

$1.00(1.00-1.00)$

${ }^{\mathrm{a}}$ RxRisk-V and Elixhauser have ten conditions in common

${ }^{\mathrm{b}} \mathrm{RxR}$ Risk-V and Charlson have six conditions in common

'Elixhauser and Charlson have 12 conditions in common, six where the diagnoses codes are identical (only 11/5 are shown because no cases of HIV/AIDS were identified in this sample)

conditions) for the condition of diabetes. For all other conditions, the agreement was weak. This agrees with previous comparison of the Charlson and RxRisk-V in a cohort of older non-joint arthroplasty Australian veterans [38]. Because of this lack of agreement amongst measurements, it is necessary to understand what measurements are used by different studies. A study using only diagnoses based measures to identify congestive heart failure, hypertension, or depression will underestimate these diagnoses, while a study using only RxRisk-V will underestimate renal disease/failure, dementia, and alcohol abuse. Underestimating these co-morbidities can impact study estimates. Utilizing validated measures is also recommended to insure that comparisons with other study results are possible. 
In addition to the differences in prevalence estimates of co-morbidities, there are further considerations for choosing the appropriate measure for co-morbidity ascertainment. Specifically, within the 64 co-morbidities identified by all measures used in this study, 28 were only identified by the RxRisk-V, 11 only by the Elixhauser and two only by the Charlson. Studies requiring the nutritional/obesity related co-morbidities such as obesity, blood loss anaemia, deficiency anaemia, fluid and electrolyte disorders, and weight loss should use the Elixhauser measure. Studies that require detail on the specific cardiovascular disease a patient is actually being treated for (e.g. anticoagulation disorder, hyperlipidaemia, ischemic heart disease/angina, and ischemic heart disease/hypertension) should use the RxRisk-V for obtaining this information. The Charlson measure should be used if history of myocardial infarction or cerebrovascular disease are needed. If a more comprehensive understanding of a patients' co-morbidity profile is necessary, we suggest using all the co-morbidity indices for both the inclusion of a greater number of conditions and a likely greater sensitivity in identifying certain conditions and provide greater amount of information to conduct case mix adjustment.

This study has several limitations. Information bias due to our use of administrative data, which can suffer from coding errors, missing data, linkage problems, and lack of detailed clinical information, was possible. Additionally, because of our sampling frame the prevalence of co-morbidities in our study may not be representative of the greater population undergoing joint arthroplasty in Australia or in other countries, where the median age of TKA and THA cohorts is between 65 and 70 years old. Ours is a sample of patients who are members of the Australian DVA system, an older patient population due to how their benefits are granted. Due to their older age we would expect them to have more comorbidities than younger arthroplasty cohorts. However, they are representative of an increasingly greater number of patients undergoing joint arthroplasty later in life and therefore offer valuable information in regards to this specific demographic.

Our study strengths included the utilisation of a captive membership population with a comprehensive database of prescription medications dispensed to its members. Due to the nature of DVA services payments, all the hospitalisation and prescriptions our cohort of patients obtains within Australia is captured. A further strength of our study is that a previous validation study has shown acceptable results in using the RxRisk-V in the Australian population in identifying co-morbidities as compared to selfreported conditions [25]. Finally, all patients in our study cohort have unique identifiers, minimizing the likelihood of data handling bias when linking their hospitalisation, demographic, and hospitalisation information.

\section{Conclusion}

Co-morbidity measures allow us to efficiently evaluate the disease burden of large cohorts of patients using existing data, such as administrative encounter and pharmacy dispensing records. Our study, along with others [38, 39] shows that the prescription based RxRisk-V measure and diagnostic based Charlson and Elixhauser measures identify a different prevalence of disease for the same conditions and have little agreement amongst them (with the exception of diabetes). Some conditions were better detected using prescription medication monitoring, while others were detected using previously inputted diagnostic codes. The specific co-morbidity measure should be chosen based on conditions necessary for that particular study, the acceptable or desirable degree of sensitivity or specificity in identifying these co-morbidities, and with the understanding of limitations involved with each of the specific measures.

\section{Abbreviations \\ AHRQ: Agency for Healthcare Research and Quality; DVA: Department of Veterans' Affairs; ICD-10-AM: International Classification of Disease, $10^{\text {th }}$ Revision, Australian Modification; IRQ: interquartile ranges; SD: standard deviations; THA: total hip arthroplasty; TKA: total knee arthroplasty; US: United States; K: kappa.}

\section{Competing interests}

All of the authors declare non-financial competing interests.

\section{Authors' contributions}

MCSI: Worked on the conception and designed the study, conducted the data analysis, conducted the interpretation of the results, and drafted and finalized the manuscript. NLP: Worked on the conception of the study, acquired data for the study, assisted with the data analysis and interpretations of the results, and provided critical review of the manuscript. EER: Worked on the conception of the study, acquired data for the study, assisted with the interpretations of the results, and provided critical review of the manuscript. SEG: Worked on the conception of the study, assisted with the interpretations of the results, and provided critical review of the manuscript. All authors read and approved the final manuscript and agree to be accountable for all aspects of the work in ensuring that questions related to the accuracy or integrity of any part of the work are appropriately investigated and resolved.

\section{Acknowledgements}

We acknowledge the provision of data for this study by the Australian Government Department of Veterans' Affairs (DVA). The DVA reviewed the manuscript to be submitted for publication but played no role in the analysis or interpretation of the data or in the preparation of this manuscript. This work was supported by an Australian Government National Health and Medical Research Council (NHMRC) Centre of Research Excellence in Post-Marketing Surveillance of Medicines and Medical Devices grant (GNT1040938). Nicole L. Pratt is supported by an NHMRC Early Career Fellowship (GNT1035889). No financial support or other benefits from commercial sources was received by any of the authors for the work reported on in the manuscript.

\section{Author details}

${ }^{1}$ Quality Use of Medicines and Pharmacy Research Centre, Medicine and Device Surveillance Centre of Research Excellence, Sansom Institute, School of Pharmacy and Medical Sciences, University of South Australia, GPO Box 2471, Adelaide 5001, South Australia, Australia. ${ }^{2}$ Australian Orthopaedic Association, National Total Joint Replacement Registry, Level 6 Bice Building, Royal Adelaide Hospital, The University of Adelaide, Adelaide 5005, SA, Australia. 
Received: 7 October 2015 Accepted: 28 November 2015 Published online: 10 December 2015

\section{References}

1. Caughey GE, Vitry Al, Gilbert AL, Roughead EE. Prevalence of comorbidity of chronic diseases in Australia. BMC Public Health. 2008:8:221. doi:10.1186/ 1471-2458-8-221.

2. Vogeli C, Shields AE, Lee TA, Gibson TB, Marder WD, Weiss KB, et al. Multiple chronic conditions: prevalence, health consequences, and implications for quality, care management, and costs. J Gen Intern Med. 2007;22 Suppl 3: 391-5. doi:10.1007/s11606-007-0322-1.

3. Cram P, Lu X, Kaboli PJ, Vaughan-Sarrazin MS, Cai X, Wolf BR, et al. Clinical characteristics and outcomes of Medicare patients undergoing total hip arthroplasty, 1991-2008. JAMA. 2011;305(15):1560-7. doi:10. 1001/jama.2011.478.

4. Cram P, Lu X, Kates SL, Singh JA, Li Y, Wolf BR. Total knee arthroplasty volume, utilization, and outcomes among Medicare beneficiaries, 1991-2010. JAMA. 2012;308(12):1227-36. doi:10.1001/2012.jama.11153.

5. Bozic KJ, Lau E, Kurtz S, Ong K, Berry DJ. Patient-related risk factors for postoperative mortality and periprosthetic joint infection in medicare patients undergoing TKA. Clin Orthop Relat Res. 2012;470(1):130-7. doi:10.1007/s11999-011-2043-3.

6. Bozic KJ, Lau E, Kurtz S, Ong K, Rubash H, Vail TP, et al. Patient-related risk factors for periprosthetic joint infection and postoperative mortality following total hip arthroplasty in Medicare patients. J Bone Joint Surg Am. 2012;94(9):794-800. doi:10.2106/JBJS.K.00072

7. Bozic KJ, Lau E, Ong K, Chan V, Kurtz S, Vail TP, et al. Risk factors for early revision after primary total hip arthroplasty in medicare patients. Clin Orthop Relat Res. 2013. doi:10.1007/s11999-013-3081-9.

8. Bozic KJ, Lau E, Ong K, Chan V, Kurtz S, Vail TP, et al. Risk factors for early revision after primary TKA in Medicare patients. Clin Orthop Relat Res. 2014;472(1):232-7. doi:10.1007/s11999-013-3045-0.

9. Olthof M, Stevens M, Bulstra SK, van den Akker-Scheek I. The association between comorbidity and length of hospital stay and costs in total hip arthroplasty patients: a systematic review. J Arthroplasty. 2013. doi:10.1016/j.arth.2013.10.008.

10. Ong KL, Lau E, Suggs J, Kurtz SM, Manley MT. Risk of subsequent revision after primary and revision total joint arthroplasty. Clin Orthop Relat Res. 2010:468(11):3070-6. doi:10.1007/s11999-010-1399-0.

11. SooHoo NF, Lieberman JR, Ko CY, Zingmond DS. Factors predicting complication rates following total knee replacement. J Bone Joint Surg Am. 2006;88(3):480-5. doi:10.2106/JBJS.E.00629.

12. Mnatzaganian G, Ryan P, Norman PE, Hiller JE. Accuracy of hospital morbidity data and the performance of comorbidity scores as predictors of mortality. $J$ Clin Epidemiol. 2012;65(1):107-15. doi:10.1016/j.jclinepi.2011.03.014.

13. Bjorgul K, Novicoff WM, Saleh KJ. Evaluating comorbidities in total hip and knee arthroplasty: available instruments. J Orthop Traumatol. 2010;1 1(4):203-9. doi:10.1007/s10195-010-0115-x.

14. Klabunde CN, Warren JL, Legler JM. Assessing comorbidity using claims data: an overview. Med Care. 2002;40(8 Suppl):IV-26-35. doi:10.1097/01.MLR. 0000020936.03651.2D.

15. Sharabiani MT, Aylin P, Bottle A. Systematic review of comorbidity indices for administrative data. Med Care. 2012;50(12):1109-18. doi:10.1097/MLR. Ob013e31825f64d0.

16. Huntley AL, Johnson R, Purdy S, Valderas JM, Salisbury C. Measures of multimorbidity and morbidity burden for use in primary care and community settings: a systematic review and guide. Ann Fam Med. 2012;10(2):134-41. doi:10.1370/afm.1363.

17. Charlson ME, Pompei P, Ales KL, MacKenzie CR. A new method of classifying prognostic comorbidity in longitudinal studies: development and validation. J Chronic Dis. 1987:40(5):373-83.

18. Elixhauser A, Steiner C, Harris DR, Coffey RM. Comorbidity measures for use with administrative data. Med Care. 1998;36(1):8-27.

19. Sloan KL, Sales AE, Liu CF, Fishman P, Nichol P, Suzuki NT, et al. Construction and characteristics of the RxRisk-V: a VA-adapted pharmacybased case-mix instrument. Med Care. 2003;41(6):761-74. doi:10.1097/01. MLR.0000064641.84967.B7.

20. George J, Vuong T, Bailey MJ, Kong DC, Marriott JL, Stewart K. Development and validation of the medication-based disease burden index. Ann Pharmacother. 2006;40(4):645-50. doi:10.1345/aph.1G204.
21. Quan H, Li B, Couris CM, Fushimi K, Graham P, Hider P, et al. Updating and validating the Charlson comorbidity index and score for risk adjustment in hospital discharge abstracts using data from 6 countries. Am J Epidemiol. 2011;173(6):676-82. doi:10.1093/aje/kwq433.

22. Fishman PA, Goodman MJ, Hornbrook MC, Meenan RT, Bachman DJ, O'Keeffe Rosetti MC. Risk adjustment using automated ambulatory pharmacy data: the RxRisk model. Med Care. 2003;41(1):84-99. doi:10.1097/01.MLR.0000039830.19812.29.

23. Johnson ML, El-Serag HB, Tran TT, Hartman C, Richardson P, Abraham NS. Adapting the Rx-Risk-V for mortality prediction in outpatient populations. Med Care. 2006;44(8):793-7. doi:10.1097/01.mlr.0000218804.41758.ef.

24. Fan VS, Maciejewski ML, Liu CF, McDonell MB, Fihn SD. Comparison of risk adjustment measures based on self-report, administrative data, and pharmacy records to predict clinical outcomes. Health Serv and Outcomes Res Methodol. 2006;6(1-2):21-36.

25. Vitry A, Wong SA, Roughead EE, Ramsay E, Barratt J. Validity of medicationbased co-morbidity indices in the Australian elderly population. Aust N Z J Public Health. 2009;33(2):126-30. doi:10.1111/j.1753-6405.2009.00357.x.

26. Quan H, Sundararajan V, Halfon P, Fong A, Burnand B, Luthi JC, et al. Coding algorithms for defining comorbidities in ICD-9-CM and ICD-10 administrative data. Med Care. 2005:43(11):1130-9.

27. Story DA, Leslie K, Myles PS, Fink M, Poustie SJ, Forbes A, et al. Complications and mortality in older surgical patients in Australia and New Zealand (the REASON study): a multicentre, prospective, observational study. Anaesthesia. 2010;65(10):1022-30. doi:10.1111/j.1365-2044.2010.06478.x.

28. van Walraven C, Austin PC, Jennings A, Quan H, Forster AJ. A modification of the Elixhauser comorbidity measures into a point system for hospital death using administrative data. Med Care. 2009;47(6):626-33. doi:10.1097/MLR.0b013e31819432e5.

29. Quality AfHRa. HCUP Comorbidity Software. Healthcare Cost and Utilization Project (HCUP) Rockville, MD. 2013. http://www.hcup-us.ahrq.gov/ toolssoftware/comorbidity/comorbidity.jsp. Accessed March 6, 2014.

30. Landis JR, Koch GG. The measurement of observer agreement for categorical data. Biometrics. 1977;33(1):159-74.

31. Kapoor A, Chew P, Silliman RA, Hylek EM, Katz JN, Cabral H, et al. Venous thromboembolism after joint replacement in older male veterans with comorbidity. J Am Geriatr Soc. 2013;61(4):590-601. doi:10.1111/jgs.12161.

32. Miric A, Inacio MC, Namba RS. Can total knee arthroplasty be safely performed in patients with chronic renal disease? Acta Orthop. 2014;85(1):71-8. doi:10.3109/17453674.2013.878829.

33. Miric A, Inacio MC, Namba RS. The effect of chronic kidney disease on total hip arthroplasty. J Arthroplasty. 2014;29(6):1225-30. doi:10.1016/j.arth.2013.12.031.

34. Dy CJ, Marx RG, Bozic KJ, Pan TJ, Padgett DE, Lyman S. Risk factors for revision within 10 years of total knee arthroplasty. Clin Orthop Relat Res. 2014;472(4):1198-207. doi:10.1007/s11999-013-3416-6.

35. Dy CJ, Bozic KJ, Pan TJ, Wright TM, Padgett DE, Lyman S. Risk factors for early revision after total hip arthroplasty. Arthritis Care Res. 2013. doi:10.1002/acr.22240.

36. Jamsen E, Peltola M, Eskelinen A, Lehto MU. Comorbid diseases as predictors of survival of primary total hip and knee replacements: a nationwide register-based study of 96754 operations on patients with primary osteoarthritis. Ann Rheum Dis. 2013;72(12):1975-82. doi:10.1136/annrheumdis-2012-202064.

37. Paxton EW, Inacio MC, Khatod M, Yue EJ, Namba RS. Kaiser Permanente National Total Joint Replacement Registry: aligning operations with information technology. Clin Orthop Relat Res. 2010;468(10):2646-63. doi:10.1007/s11999-010-1463-9.

38. Lu CY, Barratt J, Vitry A, Roughead E. Charlson and Rx-Risk comorbidity indices were predictive of mortality in the Australian health care setting. J Clin Epidemiol. 2011;64(2):223-8. doi:10.1016/j.jclinepi.2010.02.015.

39. Dominick KL, Dudley TK, Coffman CJ, Bosworth HB. Comparison of three comorbidity measures for predicting health service use in patients with osteoarthritis. Arthritis Rheum. 2005;53(5):666-72. doi:10.1002/art.21440. 